\title{
Global Stabilization of the von Kármán Plate With Boundary Feedback Acting Via Bending Moments Only
}

\author{
Mary Elizabeth Bradley ${ }^{* \dagger} \quad$ Mary Ann Horn ${ }^{\dagger+}$
}

\begin{abstract}
A von Kármán system with feedback control acting through the boundary as a bending moment only is considered. In addition to establishing uniform decay rates for the solution to this model, we show that the control is robust with respect to a parameter, $\gamma$, appearing in our system. This parameter is directly proportional to the thickness of the plate and is therefore assumed to be very small, however, since it corresponds to a portion of the principal part of the differential operator, neglecting this term would significantly change the properties of the solution. To prove our solution decays uniformly independently of $\gamma$, we begin by using multiplier methods to find preliminary energy estimates. Next, we apply "sharp" trace regularity results to obtain the desired energy estimate, modulo traces of the von Kármán nonlinearity and lower order terms. These terms are absorbed by an appropriately developed nonlinear compactness/uniqueness argument. Finally, application of nonlinear semigroup theory completes the proof.
\end{abstract}

\section{Introduction}

\subsection{Statement of the Problem}

Let $\Omega$ be an open bounded domain in $R^{2}$ with a sufficiently smooth boundary, $\Gamma$. In $\Omega$, we consider the following von Kármán system in the variables $w(t, x)$ and $\chi(w(t, x))$ with homogeneous Dirichlet boundary conditions and a control, $u$, acting through a second order boundary condition (as a moment):

$$
\begin{array}{r}
w_{t t}-\gamma^{2} \Delta w_{t t}+\Delta^{2} w+b(x) w_{t}=[w, \chi(w)] \\
w(0, \cdot)=w_{0} \\
\left.w_{t}(0, \cdot)=w_{1}\right\} \\
w=0
\end{array} \quad \begin{gathered}
\text { in } Q_{T}=(0, T) \times \Omega \\
\text { on } \Sigma_{T}=(0, T) \times \Gamma \\
\Delta w+(1-\mu) B w=-\frac{\partial}{\partial \nu} w_{t} \in L_{2}\left(\Sigma_{T}\right)
\end{gathered}
$$

*Department of Mathematics, University of Louisville, Louisville, Kentucky 40292

$\dagger$ Institute for Mathematics and its Applications, University of Minnesota, Minneapolis, Minnesota 55455

† This material is based upon work partially supported under a National Science Foundation Mathematical Sciences Postdoctoral Research Fellowship. 
where we assume $0<\gamma^{2}<M$. This parameter, $\gamma$, is proportional to the thickness of the plate and is therefore assumed to be small. Additionally, we assume $b(x) \in L^{\infty}(\Omega)$ satisfies $b(x)>0$ a.e. in $\Omega, 0<\mu<\frac{1}{2}$ is Poisson's ratio. The operator $B$ is given by

$$
B w=-\frac{\partial^{2}}{\partial \tau^{2}} w-k \frac{\partial}{\partial \nu} w=-k \frac{\partial}{\partial \nu} w
$$

where $k$ is the geodesic curvature of the boundary and the second equality follows from the homogeneous Dirichlet boundary condition.

The nonlinear term on the right-hand side of (1.1), $\chi(w)$, satisfies the system of equations

$$
\left.\begin{array}{rc}
\Delta^{2} \chi=-[w, w] & \text { in } \Omega \\
\chi=\frac{\partial}{\partial \nu} \chi=0 & \text { on } \Gamma,
\end{array}\right\}
$$

where

$$
[\phi, \psi]=\frac{\partial^{2} \phi}{\partial x^{2}} \frac{\partial^{2} \psi}{\partial y^{2}}+\frac{\partial^{2} \phi}{\partial y^{2}} \frac{\partial^{2} \psi}{\partial x^{2}}-2 \frac{\partial^{2} \phi}{\partial x \partial y} \frac{\partial^{2} \psi}{\partial x \partial y}
$$

The energy functional is given by

$$
E(t)=\frac{1}{2} \int_{\Omega}\left\{\left|w_{t}\right|^{2}+\gamma^{2}\left|\nabla w_{t}\right|^{2}+|\Delta w|^{2}+|\Delta \chi(w)|^{2}\right\} d \Omega \equiv E_{1}(t)+E_{2}(t),
$$

where $E_{2}(t)$ is defined by

$$
E_{2}(t) \equiv \frac{1}{2} \int_{\Omega}|\Delta \chi(w)|^{2} d \Omega
$$

Note that $E_{1}(t)$ represents the linear part of the energy, while $E_{2}(t)$ denotes the nonlinear part. In view of (1.5), the associated space of finite energy is $\mathcal{H} \equiv H^{2}(\Omega) \cap H_{0}^{1}(\Omega) \times H_{0}^{1}(\Omega)$, with the norm

$$
\left\|\left(w, w_{t}\right)\right\|_{\mathcal{H}}^{2} \equiv\|w\|_{H^{2}(\Omega) \cap H_{0}^{1}(\Omega)}^{2}+\left\|w_{t}\right\|_{L_{2}(\Omega)}^{2}+\gamma\left\|\nabla w_{t}\right\|_{L_{2}(\Omega)}^{2} .
$$

Our goal is to show that the boundary control, $-\frac{\partial}{\partial \nu} w_{t}$, causes the energy of our system, (1.5), to decay uniformly with respect to the initial energy as time increases. In addition, we plan to show that this control also stabilizes the limit solution when $\gamma \rightarrow 0$ in some appropriate topology.

Remark 1: We note that when $\gamma=0$, there may not be a unique solution to (1.1). Thus, our goal is show that if $w_{\gamma}$ is the solution to (1.1) when $\gamma \neq 0$, then our choice of control also stabilizes $w \equiv \lim _{\gamma \rightarrow 0} w_{\gamma}$. 


\subsection{Literature}

Although much attention has been focused on the problems of stabilization and controllability for the von Kármán plate in recent years, to our knowledge, no results deal with the boundary conditions (1.1.c) and (1.1.d), i.e., the boundary conditions which represent a simply supported plate. Local controllability and stabilization for the von Kármán plate with higher-order boundary conditions has been established in [?,?,?]. These local stabilization results gave rise to the question of global decay rates. Initial results to the question of global stabilization can be found in [?], where it was proven that, for the von Kármán model without rotational inertia or viscous damping (i.e., setting $\gamma=0$ and $b=0$ ), the energy of the resulting system is exponentially stable, provided $\Omega$ is star-shaped. More general results which include rotational moments of inertia $(\gamma \neq 0)$ can be found in [?], where global stability is proven assuming that only a portion of the boundary is available for control actions and which requires no geometric conditions on the controlled portion of the boundary.

In addition to work done on the von Kármán plate, much work has been done in the linear case, i.e., for the Kirchhoff and Euler-Bernoulli plates, with simply supported boundary conditions. In particular, uniform stabilization of the Euler-Bernoulli plate when feedback control acts via bending moments only is shown to hold in [?], assuming that only a portion of the boundary is available for control actions and requiring no geometric conditions on the controlled portion of the boundary. Also, uniform stabilization of the Kirchhoff plate with no geometric restrictions on the domain was established in [?]. Additionally, [?] proved that the feedback control for the Kirchhoff plate was robust with respect to $\gamma$, i.e., it also uniformly stabilized the corresponding limit problem in an appropriate topology when $\gamma=0$.

Our main contributions in this paper are the following:

(i) We consider the von Kármán plate with simply supported boundary conditions;

(ii) we include both rotational moments of inertia $(\gamma \neq 0)$ and light internal damping $(b \neq 0)$;

(iii) we do not impose any geometric conditions on the domain;

(iv) we establish the robustness of the control with respect to $\gamma$. 
Achieving these goals creates major technical difficulties. For example, the approach used for different plate models in [?], [?], [?], and [?] is based upon the construction of a Lyapunov function and is not readily applicable to our problem. Boundary terms (related to $\chi(w)$ ) and unstructured lower order terms appearing in needed estimates make it impossible to prove a desired differential inequality for the Lyapunov function.

Only recently have techniques been developed that can be adapted to the problem of stabilizing (1.1) with constraints (iii) and (iv). To prove our desired results, we propose a technique based on the proofs found in [?] and [?]. Among other things, the results of [?] allow us to develop a nonlinear compactness/uniqueness argument, appropriately adapted for the changes created by the new boundary conditions. This allows us to "absorb" nonlinear boundary traces and lower order terms arising from energy estimates. To establish the validity of (iii) and (iv), we will use microlocal analysis, as in [?], to prove "sharp" regularity results for the traces of the solution, however, the argument in [?] will need to be adapted to deal with the von Kármán nonlinearity.

\subsection{Statement of Main Results}

We begin by stating a well-posedness result for (1.1) which has been proven in [?].

Theorem 1.1 (Well-posedness) (i) Given initial data $\left(w_{0}, w_{1}\right) \in \mathcal{H}$, there exists a unique solution $\left(w, w_{t}\right)$ to (1.1) with

$$
w \in C\left([0, \infty) ; H^{2}(\Omega) \cap H_{0}^{1}(\Omega)\right), \quad w_{t} \in C\left([0, \infty) ; H_{0}^{1}(\Omega)\right) .
$$

(ii) Given initial data $\left\{\left(w_{0}, w_{1}\right) \in H^{4}(\Omega) \times H^{3}(\Omega):\left.w_{0}\right|_{\Gamma}=\left.w_{1}\right|_{\Gamma}=0, \Delta w_{0}+\left.(1-\mu) B w_{0}\right|_{\Gamma}=-\frac{\partial}{\partial \nu} w_{1}\right\}$, then the solution $\left(w, w_{t}\right)$ to (1.1) satisfies

$$
\begin{array}{r}
w \in C\left([0, T] ; H^{4}(\Omega) \cap H_{0}^{1}(\Omega)\right), \\
w_{t} \in C\left([0, T] ; H^{3}(\Omega) \cap H_{0}^{1}(\Omega)\right), \\
\text { and } \quad w_{t t} \in C\left([0, T] ; H^{2}(\Omega) \cap H_{0}^{1}(\Omega)\right) .
\end{array}
$$

We now state the main results of this paper. 
Theorem 1.2 Assume that the domain $\Omega \subset R^{2}$ has a sufficiently smooth boundary, $\Gamma$. Then for any initial data, $\left(w_{0}, w_{1}\right) \in \mathcal{H}$, there exist constants $C$ and $\alpha=\alpha\left(\left\|w_{0}\right\|_{H^{2}(\Omega) \cap H_{0}^{1}(\Omega)},\left\|w_{1}\right\|_{H_{0}^{1}(\Omega)}\right)$ such that the solution, $\left(w, w_{t}\right)$ of (1.1) satisfies

$$
E(t) \leq C e^{-\alpha t} E(0)
$$

Here the constant $C$ depends on the size of the initial data.

Corollary 1.1 Assume that the domain $\Omega \subset R^{2}$ has a sufficiently smooth boundary, $\Gamma$. Then for any initial data, $\left(w_{0}, w_{1}\right) \in H^{2}(\Omega) \cap H_{0}^{1}(\Omega) \times L_{2}(\Omega)$, there exist constants $C$ and $\alpha=\alpha\left(\left\|w_{0}\right\|_{H^{2}(\Omega) \cap H_{0}^{1}(\Omega)},\left\|w_{1}\right\| L_{L_{2}(\Omega)}\right)$ such that $w=\lim _{\gamma \rightarrow 0} w_{\gamma}$, where $w_{\gamma}$ is the solution of (1.1) corresponding to $\gamma \neq 0$, satisfies

$$
E_{\gamma=0}(t) \leq C e^{-\alpha t} E_{\gamma=0}(0)
$$

As before, the constant $C$ depends on the size of the initial data.

Remark 2: As in [?], the "light" internal damping, modeled by $b(x) w_{t}$, plays a key role in the stabilization of this plate. Alone it is not sufficient to uniformly stabilize the model. However, without this term, there is no apparent way to show that the boundary feedback alone uniformly stabilizes the model, since this mild damping plays a critical role in the proof of the compactness/uniqueness argument. Additionally, since most materials posess some degree of interior damping, this light damping term is physically motivated.

\section{Proof of Theorem 1.2}

To prove Theorem 1.2, we will proceed as follows. First, we begin by using multiplier methods to find preliminary energy estimates. Next, we apply "sharp" trace regularity results to obtain the desired energy estimate, modulo traces of the nonlinear function $\chi(w)$ and lower order terms. These terms will be absorbed by an appropriately developed nonlinear compactness/uniqueness argument. Finally, an argument from nonlinear semigroup theory will complete the proof. 


\subsection{Energy Estimates}

Our goal is to show that the following lemma holds.

Lemma 2.1 Let $\left(w_{0}, w_{1}\right) \in \mathcal{H}$. Then for $T$ sufficiently large, the energy of model (1.1), as given in (1.5), satisfies the following estimate

$$
E(T) \leq C(E(0))\left(1+\gamma^{2}\right)\left\{\left\|\frac{\partial}{\partial \nu} w_{t}\right\|_{L_{2}\left(\Sigma_{T}\right)}^{2}+\int_{Q_{T}} b(x) w_{t}^{2} d Q\right\}
$$

where the function $C(E(0))$ is increasing in $E(0)$ and independent of $\gamma$.

We begin by noting that the energy of model (1.1) is nonincreasing. To show this is true, we use the multiplier $w_{t}$ with equation (1.1), and integrate over $Q_{t}$ to find

$$
E(t)+2 \int_{0}^{t}\left\|\frac{\partial}{\partial \nu} w_{t}\right\|_{L_{2}(\Gamma)}^{2} d t+2 \int_{0}^{t} \int_{\Omega} b(x) w_{t}^{2}(t) d \Omega d t=E(0)
$$

\section{$2.2 \quad$ Preliminary Energy Estimates}

The first preliminary energy estimate we will prove is stated in the following proposition.

Proposition 2.1 Let $\left(w_{0}, w_{1}\right) \in \mathcal{H}$. Then the energy of model (1.1), as given in (1.5), satisfies the following estimate

$$
\begin{aligned}
{\left[T-C\left(1+\gamma^{2}\right)\right] E(T) \leq } & C_{1}\left(1+\gamma^{2}\right)\left\|\frac{\partial}{\partial \nu} w_{t}\right\|_{L_{2}\left(\Sigma_{T}\right)}^{2}+C_{2} \int_{Q_{T}} b(x) w_{t}^{2} d Q \\
& +C_{3} E^{2}(0) \int_{\Sigma_{T}}|\Delta \chi(w)| d \Gamma d t+C_{4} E^{2}(0) \int_{0}^{T}\|\chi(w)\|_{H^{3-\epsilon_{1}(\Omega)}} d t \\
& +C_{5}\left(1+\gamma^{2}\right)\|w\|_{L_{2}\left(0, T ; H^{2-\epsilon}(\Omega)\right)}^{2}+C_{6}\left\|w_{t}\right\|_{L_{2}\left(0, T ; H^{-1 / 2+\epsilon}(\Omega)\right)}^{2} .
\end{aligned}
$$

Proof: Step 1: Multiplier Identities. Consider the multiplier $w$. Note that ([?], Lemma 5.2.1)

$$
\int_{Q_{T}}[w, \chi] w d Q=\int_{Q_{T}}[w, w] \chi d Q=-\int_{Q_{T}} \Delta^{2} \chi \cdot \chi d Q=-\int_{Q_{T}}(\Delta \chi)^{2} d Q
$$

Hence, by multiplying equation (1.1) by $w$ and integrating by parts, we find

$$
\begin{aligned}
\int_{Q_{T}}\left\{w_{t}^{2}\right. & \left.+\gamma^{2}\left|\nabla w_{t}\right|^{2}\right\} d Q-\int_{Q_{T}}(\Delta w)^{2} d Q-\int_{Q_{T}}(\Delta \chi)^{2} d Q \\
& =\left.\left(w_{t}, w\right)_{\Omega}\right|_{0} ^{T}+\left.\gamma^{2}\left(\nabla w_{t}, \nabla w\right)_{\Omega}\right|_{0} ^{T}+\left.\frac{1}{2}(b(x) w, w)_{\Omega}\right|_{0} ^{T}+\left.\frac{1}{2}\left(\frac{\partial}{\partial \nu} w, \frac{\partial}{\partial \nu} w\right)_{\Gamma}\right|_{0} ^{T} .
\end{aligned}
$$


A second multiplier, $h \cdot \nabla w$, where $h \equiv x-x_{0}$ for some $x_{0} \in R^{2}$, together with equation (1.1) gives us

$$
\begin{aligned}
\int_{Q_{T}}[w, \chi](h \cdot \nabla w) d Q= & \left.(w, h \cdot \nabla w)_{\Omega}\right|_{0} ^{T}-\left.\gamma^{2}\left(\Delta w_{t}, h \cdot \nabla w\right)_{\Omega}\right|_{0} ^{T} \\
& +\int_{Q_{T}} w_{t}^{2} d Q+\int_{Q_{T}}(\Delta w)^{2} d Q+\int_{Q_{T}} b(x) w t h \cdot \nabla w d Q \\
& +\gamma^{2}\left(\Delta w_{t}, h \cdot \nabla w_{t}\right)_{Q_{T}}+\frac{1}{2} \int_{\Sigma_{T}}(\Delta w)^{2} h \cdot \nu d \Gamma d t \\
& -\int_{\Sigma_{T}} \Delta w \frac{\partial}{\partial \nu}(h \cdot \nabla w) d \Gamma d t+\int_{\Sigma_{T}}\left(\frac{\partial}{\partial \nu} \Delta w\right) h \cdot \nabla w d \Gamma d t .
\end{aligned}
$$

To simplify the above equation, we need the following two identities. From [?] (p. 115), we have

$$
\int_{Q_{T}}[w, \chi] h \cdot \nabla w d Q=-\frac{1}{2} \int_{Q_{T}}(\Delta \chi)^{2} d Q-\frac{1}{2} \int_{\Sigma_{T}}(\Delta \chi)^{2} h \cdot \nu d \Gamma d t
$$

and from [?], since $h \equiv x-x_{0}$,

$$
\left(\Delta w_{t}, h \cdot \nabla w_{t}\right)_{Q_{T}}=\left(\frac{\partial}{\partial \nu} w_{t}, h \cdot \nabla w_{t}\right)_{\Sigma_{T}}-\frac{1}{2}\left(\frac{\partial}{\partial \nu} w_{t},\left(\frac{\partial}{\partial \nu} w_{t}\right) h \cdot \nu\right)_{\Sigma_{T}}
$$

Substituting (2.7) and (2.8) into (2.6), we find

$$
\begin{aligned}
\left\|w_{t}\right\|_{L_{2}\left(Q_{T}\right)}^{2}+ & \|\Delta w\|_{L_{2}\left(Q_{T}\right)}^{2}+\frac{1}{2} \int_{Q_{T}}(\Delta \chi)^{2} d Q \\
= & -\left.(w, h \cdot \nabla w)_{\Omega}\right|_{0} ^{T}+\left.\gamma^{2}\left(\Delta w_{t}, h \cdot \nabla w\right)_{\Omega}\right|_{0} ^{T}-\frac{1}{2} \int_{\Sigma_{T}}(\Delta \chi)^{2} h \cdot \nu d \Gamma d t \\
& \quad-\gamma^{2}\left(\frac{\partial}{\partial \nu} w_{t}, h \cdot \nabla w_{t}\right)_{\Sigma_{T}}+\frac{\gamma^{2}}{2} \int_{\Sigma_{T}}\left(\frac{\partial}{\partial \nu} w_{t}\right)^{2} h \cdot \nu d \Gamma d t-\frac{1}{2} \int_{\Sigma_{T}}(\Delta w)^{2} h \cdot \nu d \Gamma d t \\
& \quad+\int_{\Sigma_{T}} \Delta w \frac{\partial}{\partial \nu}(h \cdot \nabla w) d \Gamma d t-\int_{\Sigma_{T}}\left(\frac{\partial}{\partial \nu} \Delta w\right) h \cdot \nabla w d \Gamma d t-\int_{Q_{T}} b(x) w_{t} h \cdot \nabla w d Q .
\end{aligned}
$$

Combining (2.5) with (2.9) and taking advantage of the fact that the left-hand side of (2.9) is nonnegative, we obtain

$$
\begin{aligned}
\frac{1}{4} \int_{0}^{T} E(t) d t \leq & \left.\frac{1}{4}\left(w_{t}, w\right)_{\Omega}\right|_{0} ^{T}+\left.\frac{\gamma^{2}}{4}\left(\nabla w_{t}, \nabla w\right)_{\Omega}\right|_{0} ^{T}+\left.\frac{1}{8}(b(x) w, w)_{\Omega}\right|_{0} ^{T}+\left.\frac{1}{4}\left(\frac{\partial}{\partial \nu} w, \frac{\partial}{\partial \nu} w\right)_{\Gamma}\right|_{0} ^{T} \\
& -\left.(w, h \cdot \nabla w)_{\Omega}\right|_{0} ^{T}+\left.\gamma^{2}\left(\Delta w_{t}, h \cdot \nabla w\right)_{\Omega}\right|_{0} ^{T}-\frac{1}{2} \int_{\Sigma_{T}}(\Delta \chi)^{2} h \cdot \nu d \Gamma d t \\
& -\gamma^{2}\left(\frac{\partial}{\partial \nu} w_{t}, h \cdot \nabla w_{t}\right)_{\Sigma_{T}}+\frac{\gamma^{2}}{2} \int_{\Sigma_{T}}\left(\frac{\partial}{\partial \nu} w_{t}\right)^{2} h \cdot \nu d \Gamma d t-\frac{1}{2} \int_{\Sigma_{T}}(\Delta w)^{2} h \cdot \nu d \Gamma d t \\
& +\int_{\Sigma_{T}} \Delta w \frac{\partial}{\partial \nu}(h \cdot \nabla w) d \Gamma d t-\int_{\Sigma_{T}}\left(\frac{\partial}{\partial \nu} \Delta w\right) h \cdot \nabla w d \Gamma d t-\int_{Q_{T}} b(x) w_{t} h \cdot \nabla w d Q .
\end{aligned}
$$

Step 2: Bounding Terms. Our next step is to bound any undesired terms on the right-hand side of (2.10) by combinations of the norm of our control and the energy of the system. We can bound the terms evaluated at 0 and $T$ as follows:

$$
\begin{gathered}
C_{1}\left(1+\gamma^{2}\right) E(T)+C_{2}\left(1+\gamma^{2}\right) E(0) \geq \\
\geq\left.\left.\frac{1}{4}\left|\left(w_{t}, w\right)_{\Omega}\right|_{0}^{T}\left|+\frac{\gamma^{2}}{4}\right|\left(\nabla w_{t}, \nabla w\right)_{\Omega}\right|_{0} ^{T}\left|+\frac{1}{4}\right|\left(\frac{\partial}{\partial \nu} w, \frac{\partial}{\partial \nu} w\right)_{\Gamma}\right|_{0} ^{T} \mid \\
+\left.\left|(w, h \cdot \nabla w)_{\Omega}\right|_{0}^{T}\left|+\gamma^{2}\right|\left(\Delta w_{t}, h \cdot \nabla w\right)_{\Omega}\right|_{0} ^{T} \mid
\end{gathered}
$$


and

$$
\begin{aligned}
\left|(b(x) w, w)_{\Omega}\right|_{0}^{T} \mid & \leq \tilde{C} \int_{\Omega}\left[w^{2}(T)+w^{2}(0)\right] d \Omega \\
& \leq C\{E(T)+E(0)\}
\end{aligned}
$$

since $b \in L^{\infty}(\Omega)$.

Next, the boundary terms on the third line of (2.10) may be bounded straightforwardly. Since $\left.w\right|_{\Sigma_{T}}=0$,

$$
\gamma^{2}\left|\left(\frac{\partial}{\partial \nu} w_{t}, h \cdot \nabla w_{t}\right)_{\Sigma_{T}}\right|+\frac{\gamma^{2}}{2}\left|\int_{\Sigma_{T}}\left(\frac{\partial}{\partial \nu} w_{t}\right)^{2} h \cdot \nu d \Gamma d t\right| \leq \gamma^{2} C\left\|\frac{\partial}{\partial \nu} w_{t}\right\|_{L_{2}\left(\Sigma_{T}\right)}^{2} .
$$

Using boundary condition (1.1.d) and standard trace theory, we find

$$
\begin{aligned}
\left|\int_{\Sigma_{T}}(\Delta w)^{2} h \cdot \nu d \Gamma d t\right| & \leq M_{k}\left\|\frac{\partial}{\partial \nu} w\right\|_{L_{2}\left(\Sigma_{T}\right)}^{2}+C\left\|\frac{\partial}{\partial \nu} w_{t}\right\|_{L_{2}\left(\Sigma_{T}\right)}^{2} \\
& \leq M_{k}\|w\|_{L_{2}\left(0, T ; H^{2-\epsilon}(\Omega)\right)}^{2}+C\left\|\frac{\partial}{\partial \nu} w_{t}\right\|_{L_{2}\left(\Sigma_{T}\right)}^{2} .
\end{aligned}
$$

Applying Hölder's inequality to the last term on the right-hand side of (2.10), we obtain

$$
\begin{aligned}
\left|\int_{Q_{T}} b(x) w_{t} h \cdot \nabla w d Q\right| & \leq C_{1} \int_{Q_{T}} b(x) w_{t}^{2} d Q+C_{2}\|\nabla w\|_{L_{2}\left(Q_{T}\right)}^{2} \\
& \leq C_{1} \int_{Q_{T}} b(x) w_{t}^{2} d Q+C_{2}\|w\|_{L_{2}\left(0, T ; H^{2-\epsilon}(\Omega)\right)}^{2}
\end{aligned}
$$

since $b \in L^{\infty}(\Omega)$.

Substituting all of the above bounds, (2.11)-(2.15), into (2.10) and recalling that the energy of our system is nonincreasing, we arrive at

$$
\begin{aligned}
\int_{0}^{T} E(t) d t-C\left(1+\gamma^{2}\right) E(0) \leq & C_{1}\left(1+\gamma^{2}\right)\left\|\frac{\partial}{\partial \nu} w_{t}\right\|_{L_{2}\left(\Sigma_{T}\right)}^{2}+C_{2} \int_{Q_{T}} b(x) w_{t}^{2} d Q \\
& +C_{3} \int_{\Sigma_{T}}(\Delta \chi)^{2} d \Gamma d t+C_{4}\|w\|_{L_{2}\left(0, T ; H^{2-\epsilon}(\Omega)\right)}^{2} \\
& +\int_{\Sigma_{T}} \Delta w \frac{\partial}{\partial \nu}(h \cdot \nabla w) d \Gamma d t-\int_{\Sigma_{T}}\left(\frac{\partial}{\partial \nu} \Delta w\right) h \cdot \nabla w d \Gamma d t .
\end{aligned}
$$

To bound the nonlinear term on the right-hand side of (2.16), we apply a result from [?], which gives us

$$
\int_{\Sigma_{T}}|\Delta \chi|^{2} d \Gamma d t \leq \epsilon C E^{2}(0) \int_{0}^{T} E(t) d t+\frac{1}{4 \epsilon} \int_{\Sigma_{T}}|\Delta \chi(w)| d \Gamma d t
$$

Setting $\epsilon=\frac{1}{2 C_{3} C E^{2}(0)}$ and substituting the result into (2.16), we obtain the estimate:

$$
\begin{aligned}
\frac{1}{2} \int_{0}^{T} E(t) d t-C\left(1+\gamma^{2}\right) E(0) \leq & C_{1}\left(1+\gamma^{2}\right)\left\|\frac{\partial}{\partial \nu} w_{t}\right\|_{L_{2}\left(\Sigma_{T}\right)}^{2}+C_{2} \int_{Q_{T}} b(x) w_{t}^{2} d Q \\
& +C_{3} E^{2}(0) \int_{\Sigma_{T}}|\Delta \chi(w)| d \Gamma d t+C_{4}\|w\|_{L_{2}\left(0, T ; H^{2-\epsilon}(\Omega)\right)}^{2} \\
& +\int_{\Sigma_{T}} \Delta w \frac{\partial}{\partial \nu}(h \cdot \nabla w) d \Gamma d t-\int_{\Sigma_{T}}\left(\frac{\partial}{\partial \nu} \Delta w\right) h \cdot \nabla w d \Gamma d t .
\end{aligned}
$$


Step 3: Bounding Integral Terms. Our next step is to find bounds for the two integral terms in (2.18). We bound the first integral term on the last line of (2.18) by using a microlocal analysis result which can be found in [?] (see Proposition 3.1). Application of this proposition to our problem gives us the following lemma.

Lemma 2.2 Let $w$ be the solution to (1.1) and let $0<\alpha<\frac{T}{2}$. Then $w$ satisfies the following inequality.

$$
\left\|\frac{\partial}{\partial \tau} \frac{\partial}{\partial \nu} w\right\|_{L_{2}(\alpha, T-\alpha ; \Gamma)}^{2} \leq C\left\{\left\|\frac{\partial}{\partial \nu} w_{t}\right\|_{L_{2}\left(\Sigma_{T}\right)}^{2}+\|f\|_{H^{-3 / 2+\epsilon}\left(Q_{T}\right)}^{2}+\left(1+\gamma^{2}\right)\|w\|_{L_{2}\left(0, T ; H^{2-\epsilon}(\Omega)\right)}^{2}\right\}
$$

where $0<\epsilon<\frac{1}{2}$, the constant $C$ does not depend on $\gamma>0$ and $f(x, t)=-b(x) w_{t}+[\chi(w), w]$.

To bound the second integral term on the last line of (2.18), we apply a generalization of a result in [?] (see Lemma 2.3), which is stated in the following lemma.

Lemma 2.3 Let $w$ be the solution to (1.1) and let $\alpha$ and $\epsilon$ be as in Lemma 2.D. Then $w$ satisfies the following inequality.

$$
\begin{gathered}
\left\|\frac{\partial}{\partial \nu} \Delta w\right\|_{H^{-1}\left(\alpha, T-\alpha ; L_{2}(\Gamma)\right)}^{2} \leq C_{T}\left(1+\gamma^{2}\right)\left\|\frac{\partial}{\partial \nu} w_{t}\right\|_{L_{2}\left(\Sigma_{T}\right)}^{2}+C(T+1)\left(1+\gamma^{2}\right)\|w\|_{L_{2}\left(0, T ; H^{2-\epsilon}(\Omega)\right)}^{2} \\
+C\|f\|_{L_{2}\left(0, T ; H^{-1 / 2+\epsilon}(\Omega)\right)}
\end{gathered}
$$

where $C_{T}$ and $C$ are independent of $\gamma$ and $f(x, t)=-b(x) w_{t}+[\chi(w), w]$.

Lemma 2.3 will be proven in the third section.

Applying Lemma 2.2 and Lemma 2.3 on the interval $(\alpha, T-\alpha)$ to inequality (2.18), we find

$$
\begin{aligned}
\frac{1}{2} \int_{\alpha}^{T-\alpha} E(t) d t-C\left(1+\gamma^{2}\right) E(\alpha) \leq & C_{T}\left(1+\gamma^{2}\right)\left\|\frac{\partial}{\partial \nu} w_{t}\right\|_{L_{2}\left(\Sigma_{T}\right)}^{2}+C_{2} \int_{Q_{T}} b(x) w_{t}^{2} d Q \\
& +C_{3} E^{2}(\alpha) \int_{\Sigma_{T}}|\Delta \chi(w)| d \Gamma d t+C_{4}\left(1+\gamma^{2}\right)\|w\|_{L_{2}\left(0, T ; H^{2-\epsilon}(\Omega)\right)}^{2} \\
& +C_{5}\|f\|_{L_{2}\left(0, T ; H^{-1 / 2+\epsilon}(\Omega)\right)}^{2}
\end{aligned}
$$

where $f(x, t)=-b(x) w_{t}+[\chi(w), w]$.

To bound the term corresponding to $f$, we begin as follows. First, the term associated with $b(x)$ can be bounded directly as follows:

$$
\left\|b(x) w_{t}\right\|_{L_{2}\left(0, T ; H^{-1 / 2+\epsilon}(\Omega)\right)} \leq C\left\|w_{t}\right\|_{L_{2}\left(0, T ; H^{-1 / 2+\epsilon}(\Omega)\right)},
$$


since $\left.b(x) \in L^{\infty}(\Omega)\right)$. Next, the nonlinear part of $f$ may be bounded as in [?], which tells us that for every $\epsilon_{0}>0$, there exists an $\epsilon_{1}>0$ such that

$$
\|[\chi(w), w]\|_{H^{-\epsilon_{0}(\Omega)}} \leq C\|w\|_{H^{2}(\Omega)}^{2}\|\chi(w)\|_{H^{3-\epsilon_{1}(\Omega)}}^{1 / 2} .
$$

Hence,

$$
\int_{0}^{T}\|f\|_{L_{2}\left(0, T ; H^{\left.-\epsilon_{0}(\Omega)\right)}\right.}^{2} d t \leq C\left\{\left\|w_{t}\right\|_{L_{2}\left(0, T ; H^{-1 / 2+\epsilon(\Omega))}\right.}^{2}+E^{2}(0) \int_{0}^{T}\|\chi(w)\|_{H^{3-\epsilon_{1}(\Omega)}} d t\right\}
$$

Substituting the above inequality into (2.21), we find

$$
\begin{aligned}
\frac{1}{2} \int_{\alpha}^{T-\alpha} E(t) d t-C\left(1+\gamma^{2}\right) E(\alpha) \leq & C_{T}\left(1+\gamma^{2}\right)\left\|\frac{\partial}{\partial \nu} w_{t}\right\|_{L_{2}\left(\Sigma_{T}\right)}^{2}+C_{2} \int_{Q_{T}} b(x) w_{t}^{2} d Q \\
& +C_{3} E^{2}(\alpha) \int_{\Sigma_{T}}|\Delta \chi(w)| d \Gamma d t+C_{4} E^{2}(0) \int_{0}^{T}\|\chi(w)\|_{H^{3-\epsilon_{1}(\Omega)}} d t \\
& +C_{5}\left(1+\gamma^{2}\right)\|w\|_{L_{2}\left(0, T ; H^{2-\epsilon}(\Omega)\right)}^{2}+C_{6}\left\|w_{t}\right\|_{L_{2}\left(0, T ; H^{-1 / 2+\epsilon}(\Omega)\right)}^{2} .
\end{aligned}
$$

Recalling (2.2), we know $E(\alpha) \leq E(0)$ and $E^{2}(\alpha) \leq E^{2}(0)$. Hence, by choosing $T$ to be sufficiently large, we obtain our desired inequality, (2.3).

\subsection{Compactness-Uniqueness Argument}

We have now proven that the energy of system (1.1) is bounded by the feedback controls plus lower order and nonlinear terms. To obtain the desired goal of Lemma 2.1, we must prove that the energy is bounded by the feedbacks alone. To accomplish this, we first observe that (see [?]):

$$
\int_{\Sigma}|\Delta \chi| d \Gamma d t \leq m e s(\Gamma)^{1 / 2} \int_{0}^{T}\|\Delta \chi\|_{L_{2}(\Gamma)} d t \leq C \int_{0}^{T}\|\Delta \chi\|_{H^{1 / 2+\epsilon}(\Omega)} d t \leq C \int_{0}^{T}\|\chi\|_{H^{3-\epsilon}(\Omega)} d t
$$

Thus, it suffices to prove the following lemma.

Lemma 2.4 Compactness-Uniqueness Let $\left(w, w_{t}\right)$ be a solution pair for (1.1). Then for any $\epsilon>0$,

$$
\int_{0}^{T}\|\chi(w)\|_{H^{3-\epsilon}(\Omega)} d t+\text { l.o. }(w) \leq C(E(0))\left\{\int_{\Sigma_{T}}\left|\frac{\partial}{\partial \nu} w_{t}\right|^{2} d \Gamma d t+\int_{Q_{T}} b(x) w_{t}^{2} d Q\right\},
$$

where $C(E(0))$ is independent of $\gamma$, is an increasing function of the initial energy, $E(0)$, and the lower order term are denoted by l.o.( $(w)$ which is defined to be:

$$
\text { l.o. }(w) \equiv\|w\|_{H^{2-\epsilon}(\Omega)}^{2}+\left\|w_{t}\right\|_{H^{-\epsilon}(\Omega)}^{2}
$$


Remark: Notice that the nonlinear term on the left hand side of (2.27) is not a lower order term. Indeed, its definition requires two spatial derivatives of the function $w$ which is exactly at the level of the energy functional.

We begin the proof of Lemma 2.4 with the following proposition.

Proposition 2.2 Let $\left(w, w_{t}\right)$ be a solution pair for (1.1). Then for any $\epsilon>0$,

$$
\int_{0}^{T}\|\chi(w)\|_{H^{3-\epsilon}(\Omega)} d t+\text { l.o. }(w) \leq C_{\gamma}(E(0))\left\{\int_{\Sigma_{T}}\left|\frac{\partial}{\partial \nu} w_{t}\right|^{2} d \Gamma d t+\int_{Q_{T}} b(x) w_{t}^{2} d Q\right\}
$$

where $C_{\gamma}(E(0))$ may depend on $\gamma$ and is an increasing function of the initial energy, E(0), and l.o. $(w)$ is defined by (2.28).

Proof: The proof is by contradiction. Suppose (2.27) does not hold. Then there exists a sequence of functions $\left\{w_{n}(t)\right\}$ in $\mathcal{H}$ which satisfies the system

$$
\begin{array}{cc}
w_{n}^{\prime \prime}-\gamma^{2} \Delta w_{n}^{\prime \prime}+\Delta^{2} w_{n}+b w_{n}^{\prime}=\left[w_{n}, \chi\left(w_{n}\right)\right] & \text { in } Q_{T} \\
w_{n}(0, \cdot)=w_{n 0} ; w_{n}^{\prime}(0, \cdot)=w_{n 1} & \text { in } \Omega \\
w_{n}=0 & \text { on } \Sigma_{T} \\
\Delta w_{n}+(1-\mu) B w_{n}=-\frac{\partial}{\partial \nu} w_{n}^{\prime} & \text { on } \Sigma_{T}
\end{array}
$$

and such that

$$
\lim _{n \rightarrow \infty} \frac{\operatorname{loo}\left(w_{n}\right)+\int_{0}^{T}\left\|\chi\left(w_{n}\right)\right\|_{H^{3-\epsilon}(\Omega)} d t}{\int_{\Sigma_{T}}\left|\frac{\partial}{\partial \nu} w_{n}^{\prime}\right|^{2} d \Gamma d t+\int_{Q_{T}} b\left(w_{n}^{\prime}\right)^{2}}=\infty
$$

where the initial energy (as prescribed by initial data $\left(w_{n 0}, w_{n 1}\right)$ ) is uniformly bounded $\forall n$. (Note: For convenience, we denote the time derivatives by '.)

Denoting the sequence

$$
c_{n} \equiv\left\{l . o .\left(w_{n}\right)+\int_{0}^{T}\left\|\chi\left(w_{n}\right)\right\|_{H^{3-\epsilon}(\Omega)} d t\right\}^{1 / 2}
$$

we introduce the new variable

$$
v_{n} \equiv \frac{w_{n}}{c_{n}}
$$


We observe that $v_{n}$ satisfies the system

$$
\begin{array}{lc}
v_{n}^{\prime \prime}-\gamma^{2} \Delta v_{n}^{\prime \prime}+\Delta^{2} v_{n}+b v_{n}^{\prime}=\left[v_{n}, \chi\left(w_{n}\right)\right] & \text { in } Q_{T} \\
v_{n}(0, \cdot)=v_{n 0} ; v_{n}^{\prime}(0, \cdot)=v_{n 1} & \text { in } \Omega \\
v_{n}=0 & \text { on } \Sigma_{T} \\
\Delta v_{n}+(1-\mu) B v_{n}=-\frac{\partial}{\partial \nu} v_{n}^{\prime} & \text { on } \Sigma_{T} .
\end{array}
$$

By the quadratic dependence of $\chi$ on $w_{n}$,

$$
\text { l.o. }\left(v_{n}\right)+\int_{0}^{T}\left\|\chi\left(v_{n}\right)\right\|_{H^{3-\epsilon(\Omega)}} d t=\frac{\text { l.o. }\left(w_{n}\right)+\int_{0}^{T}\left\|\chi\left(w_{n}\right)\right\|_{H^{3-\epsilon}(\Omega)} d t}{\text { l.o. }\left(w_{n}\right)+\int_{0}^{T}\left\|\chi\left(w_{n}\right)\right\|_{H^{3-\epsilon}(\Omega)} d t} \equiv 1 \text {. }
$$

Hence, by using (2.31), we see that $v_{n}$ satisfies

$$
\lim _{n \rightarrow \infty} \int_{\Sigma_{T}}\left|\frac{\partial}{\partial \nu} v_{n}^{\prime}\right|^{2} d \Gamma d t+\int_{Q_{T}} b\left(v_{n}^{\prime}\right)^{2} d \Omega d t=0
$$

By (2.36) and the boundary conditions in (2.34), we have the following convergence properties:

$$
\begin{aligned}
& \text { (i) } v_{n}^{\prime} \rightarrow 0 \text { in } L^{2}\left(Q_{T}\right) \\
& \text { (ii) } v_{n}^{\prime} \rightarrow 0 \text { in } H^{1}\left(\Sigma_{T}\right) .
\end{aligned}
$$

In order to pass with a limit on (2.34), we need first to determine further convergence properties for $v_{n}$ and for our nonlinear terms.

To determine the convergence properties of $v_{n}$, we will use the energy estimates which were derived in the previous section. If we denote the energy of $(2.34)$ by $\widetilde{E}_{n}(t)$ and the energy of system $(2.30)$ by $E_{n}(t)$, we have by Proposition 2.1, (2.26) and (2.2)

$$
\begin{aligned}
\widetilde{E}_{1 n}(0) \leq C_{T, \gamma}\left(E_{n}(0)\right)\{ & \int_{\Sigma_{T}}\left|\frac{\partial}{\partial \nu} v_{n}^{\prime}\right|^{2} d \Gamma d t+\int_{Q_{T}} b\left(v_{n}^{\prime}\right)^{2} d \Omega d t \\
& \left.+ \text { l.o. }\left(v_{n}\right)+\int_{0}^{T}\left\|\chi\left(v_{n}\right)\right\|_{H^{3-\epsilon}(\Omega)} d t\right\},
\end{aligned}
$$

where $C_{T, \gamma}\left(E_{n}(0)\right)$ possibly depends on $\gamma$ and the initial energy for the system $(2.30), E_{n}(0)$ (not on $\widetilde{E}_{n}(0)$ ) and $\widetilde{E}_{1 n}(0)$ represents the linear part of the initial energy (as in (1.5)). By using (2.36), we may assume without loss of generality that

$$
\int_{\Sigma_{T}}\left|\frac{\partial}{\partial \nu} v_{n}^{\prime}\right|^{2} d \Gamma d t+\int_{Q_{T}} b\left(v_{n}^{\prime}\right)^{2} d \Omega d t \leq 1
$$


Consequently, we have by $(2.35)$ and (2.39) that $\widetilde{E}_{1 n}(0) \leq \widetilde{C}_{T, \gamma}$ where $\widetilde{C}_{T, \gamma}$ may depend on $E_{n}(0)$. Since the $E_{n}(0)$ are uniformly bounded, we conclude that $\widetilde{E}_{1 n}(0)$ are uniformly bounded as well.

We may now use the results of our well-posedness theorem applied to (2.34) to obtain that

$$
\begin{aligned}
& \text { (i) }\left\|v_{n}\right\|_{C\left([0, T] ; H^{2}(\Omega)\right)} \leq M \\
& \text { (ii) }\left\|v_{n}^{\prime}\right\|_{C\left([0, T] ; H^{1}(\Omega)\right)} \leq M,
\end{aligned}
$$

which implies, in particular, that $\left\{v_{n}\right\}$ are uniformly bounded in $H^{1}([0, T] \times \Omega)$. Hence, by the compact Sobolev imbeddings and trace theory, we see that

$$
\begin{array}{ccc} 
& & v_{n} \stackrel{w}{\rightarrow} v \text { in } L^{2}\left([0, T] ; H^{2}(\Omega)\right) \text { and } v_{n}^{\prime} \stackrel{w}{\rightarrow} v^{\prime} \text { in } L^{2}\left([0, T] ; H^{1}(\Omega)\right) \\
\Longrightarrow & v_{n} \stackrel{w}{\rightarrow} v \text { in } H^{1}([0, T] \times \Omega) \\
\Longrightarrow & v_{n} \rightarrow v \text { in } L^{2}\left(\Sigma_{T}\right) .
\end{array}
$$

Also, since $E_{n}(0) \leq M$, the well-posedness for (1.1) yields

$$
\begin{array}{r}
w_{n} \stackrel{w}{\rightarrow} w \text { in } L_{2}\left([0, T] ; H^{2}(\Omega)\right) \\
\text { and } w_{n}^{\prime} \stackrel{w}{\rightarrow} w^{\prime} \text { in } L_{2}\left([0, T] ; H^{1}(\Omega)\right),
\end{array}
$$

so that $\left\{w_{n}\right\}$ has the same convergence properties as $\left\{v_{n}\right\}$.

We now examine the convergence properties of the von Kármán nonlinearity, $\left[v_{n}, \chi\left(w_{n}\right)\right]$. We state here three results which are proven in [?].

Lemma 2.5 Let $w_{n} \stackrel{w}{\rightarrow} w$ in $H^{2}(\Omega)$. Then $\chi\left(w_{n}\right) \stackrel{w}{\rightarrow} \chi(w)$ in $H_{0}^{2}(\Omega)$.

Lemma 2.6 Assume that

$$
\begin{gathered}
w_{n} \stackrel{w}{\rightarrow} w \text { in } L^{2}\left([0, T] ; H^{2}(\Omega)\right) \\
w_{n}^{\prime} \stackrel{w}{\rightarrow} w^{\prime} \text { in } L^{2}\left([0, T] ; H^{1}(\Omega)\right) .
\end{gathered}
$$

Then for every $\epsilon>0$,

$$
\chi\left(w_{n}\right) \rightarrow \chi(w) \text { in } C\left([0, T] ; H^{3-\epsilon}(\Omega)\right) .
$$

Lemma 2.7 Let $v_{n} \stackrel{w}{\longrightarrow} v$ in $H^{2}(\Omega)$ and assume that

$$
w_{n} \stackrel{w}{\rightarrow} w \text { in } L^{2}\left([0, T] ; H^{2}(\Omega)\right) .
$$

Then $\left[v_{n}, \chi\left(w_{n}\right)\right] \rightarrow[v, \chi(w)]$ in the sense of distributions. 
We are now in a position to pass with a limit on equation (2.34). We will consider two cases.

Case 1: $c_{0}=\left\{\text { l.o. }(w)+\int_{0}^{T}\|\chi(w)\|_{H^{3-\epsilon}(\Omega)} d t\right\}^{1 / 2} \neq 0$. By Lemmas 2.5, 2.6, and 2.7, (2.42) and (2.32), and by the compactness properties of l.o. $(w)$, we have $c_{n} \rightarrow c_{0}$, hence $v=w / c_{0}$. Using (2.37), Lemma 2.7 and passing with a limit on (2.34), we obtain the limit system

$$
\begin{array}{ll}
\Delta^{2} v=[v, \chi(w)]=\frac{1}{c_{0}}[w, \chi(w)] & \text { in } Q_{T} \\
v=0 & \text { on } \Sigma_{T} \\
\Delta v+(1-\mu) B v=0 & \text { on } \Sigma_{T} .
\end{array}
$$

Let $\mathcal{A}$ be the positive, self-adjoint operator defined by:

$$
\mathcal{A} h \equiv \Delta^{2} h, \quad \mathcal{D}(\mathcal{A})=\left\{h \in H^{4}(\Omega) \cap H_{0}^{1}(\Omega): \Delta h+\left.(1-\mu) B h\right|_{\Gamma}=0\right\} .
$$

With this definition, we can write (2.46) abstractly as:

$$
\mathcal{A} v=[v, \chi(w)]=\frac{1}{c_{0}}[w, \chi(w)]
$$

Multiplying (2.48) by $v$ and integrating over $\Omega$, we find

$$
\begin{aligned}
0 & =(\mathcal{A} v, v)_{L_{2}(\Omega)}-([v, \chi(w)], v)_{L_{2}(\Omega)} \\
& =\left\|\mathcal{A}^{1 / 2} v\right\|_{L_{2}(\Omega)}-\int_{\Omega}[v, v] \chi(w) d \Omega \\
& =\left\|\mathcal{A}^{1 / 2} v\right\|_{L_{2}(\Omega)}+\int_{\Omega} \Delta^{2} \chi(v) \chi(w) d \Omega \\
& =\left\|\mathcal{A}^{1 / 2} v\right\|_{L_{2}(\Omega)}+\frac{1}{c_{0}^{2}} \int_{\Omega}(\Delta \chi(w))^{2} d \Omega .
\end{aligned}
$$

Therefore, by the positivity of $\mathcal{A}$,

$$
v \equiv 0 \quad \text { in } Q_{T}
$$

In order to complete the contradiction (see (2.35)), we must prove

$$
\left(\text { l.o. }\left(v_{n}\right)+\int_{0}^{T}\left\|\chi\left(v_{n}\right)\right\|_{H^{3-\varepsilon_{(\Omega)}}} d t\right) \longrightarrow 0 .
$$

But this follows from the work of [?] (Lemmas 2.5 and 2.6), (2.40), (2.41) and (2.50). This provides the contradiction, and hence the proof of Lemma 2.2 for Case 1.

Case 2: $c_{0}=0$, (i.e. $\chi(w) \equiv 0$ and l.o. $(w)=0$.) In this case, we will again use the result of [?]. Here we use the fact that $\chi\left(w_{n}\right) \rightarrow 0$ in $C\left([0, T] ; H^{3-\epsilon}(\Omega)\right)$ in combination with $(2.40)$ and Lemma 2.7 in order 
to obtain that $\left[v_{n}(t), \chi\left(w_{n}\right)(t)\right] \rightarrow 0$ in $\mathcal{D}^{\prime}(\Omega)$. By using (2.37) and passing with a limit on system (2.46), we obtain

$$
\begin{array}{ll}
\Delta^{2} v=0 & \text { in } Q_{T} \\
v=0 & \text { on } \Sigma_{T} \\
\Delta v+(1-\mu) B v=0 & \text { on } \Sigma_{T} .
\end{array}
$$

The same argument as in Case 1 yields $v \equiv 0$ and

$$
\text { l.o. }\left(v_{n}\right)+\int_{0}^{T}\left\|\chi\left(w_{n}\right)\right\|_{H^{3-\epsilon}(\Omega)} d t \longrightarrow 0,
$$

which gives us the contradiction. $\square$.

Completion of the Proof of Lemma 2.4: By Proposition 2.2, we know the solution to (1.1), w, satisfies (2.29). Assume $C_{\gamma}(E(0))$ is not uniformly bounded in $\gamma$. Since $\gamma \rightarrow 0$, without loss of generality, we may assume $\gamma<1$. Then there exists a sequence of functions $\left\{w_{\gamma}(t)\right\}$ in $\mathcal{H}$ which satisfies the system

$$
\begin{array}{lc}
w_{\gamma}^{\prime \prime}-\gamma^{2} \Delta w_{\gamma}^{\prime \prime}+\Delta^{2} w_{\gamma}+b w_{\gamma}^{\prime}=\left[w_{\gamma}, \chi\left(w_{\gamma}\right)\right] & \text { in } Q_{T} \\
w_{\gamma}(0, \cdot)=w_{0} ; w_{\gamma}^{\prime}(0, \cdot)=w_{1} & \text { in } \Omega \\
w_{\gamma}=0 & \text { on } \Sigma_{T} \\
\Delta w_{\gamma}+(1-\mu) B w_{\gamma}=-\frac{\partial}{\partial \nu} w_{\gamma}^{\prime} & \text { on } \Sigma_{T}
\end{array}
$$

and such that

$$
\lim _{\gamma \rightarrow 0} \frac{l . o .\left(w_{\gamma}\right)+\int_{0}^{T}\left\|\chi\left(w_{\gamma}\right)\right\|_{H^{3-\epsilon}(\Omega)} d t}{\int_{\Sigma_{T}}\left|\frac{\partial}{\partial \nu} w_{\gamma}^{\prime}\right|^{2} d \Gamma d t+\int_{Q_{T}} b\left(w_{\gamma}^{\prime}\right)^{2}}=\infty,
$$

where the initial energy (as prescribed by initial data $\left(w_{0}, w_{1}\right)$ ) is uniformly bounded $\forall \gamma$. Normalizing as in (2.32) and (2.33), we find that $v_{\gamma}$ satisfies $(2.34),(2.35)$, and (2.36) (with $n$ replaced by $\gamma$ ).

Proceeding as in the proof of Proposition 2.2 (using wellposedness and energy estimate (2.2)), we readily obtain that

$$
\left\|w_{\gamma}\right\|_{C\left(0, T ; H^{2}(\Omega)\right)}+\left\|w_{\gamma}^{\prime}\right\|_{C\left(0, T ; L_{2}(\Omega)\right)}+\gamma\left\|w_{\gamma}^{\prime}\right\|_{C\left(0, T ; H^{1}(\Omega)\right)} \leq C .
$$

In particular, since $[0, T]$ is bounded, we have the convergence properties

$$
\left\{\begin{array}{c}
w_{\gamma} \stackrel{w}{\rightarrow} w \quad \text { in } L_{2}\left(0, T ; H^{2}(\Omega)\right) \\
w_{\gamma}^{\prime} \stackrel{w}{\rightarrow} w^{\prime} \quad \text { in } L_{2}\left(0, T ; L_{2}(\Omega)\right)
\end{array}\right.
$$


We now prove the following lemma which is analagous to Lemma 2.6.

Lemma 2.8 Assume that $w_{\gamma}$ satisfies (2.54), (2.56) and (2.57). Then for every $0<\epsilon<\frac{1}{2}$,

$$
\chi\left(w_{\gamma}\right) \longrightarrow \chi(w) \text { in } C\left(0, T ; H^{3-\epsilon}(\Omega)\right)
$$

Proof: By the results of [?], along with our own assumptions, we see that for $\epsilon>0$,

$$
\left\|\chi\left(w_{\gamma}\right)\right\|_{L_{\infty}\left(0, T ; H^{3-\epsilon}(\Omega)\right)} \leq C_{1} .
$$

We now show that

$$
\left\|\frac{\partial}{\partial t} \chi\left(w_{\gamma}\right)\right\|_{L_{2}\left(0, T ; H^{1-\epsilon}(\Omega)\right)} \leq C_{2}
$$

Observe that

$$
\frac{\partial}{\partial t} \chi\left(w_{\gamma}\right)=-2 \mathcal{K}^{-1}\left[w_{\gamma}^{\prime}, w_{\gamma}\right]
$$

where the operator $\mathcal{K}: H^{4}(\Omega) \longrightarrow L_{2}(\Omega)$ is defined by

$$
\mathcal{K} w \equiv \Delta^{2} w, \quad \mathcal{D}(\mathcal{K})=H^{4}(\Omega) \cap H_{0}^{2}(\Omega)
$$

Let $\phi \in H^{3+\epsilon}(\Omega) \cap H_{0}^{2}(\Omega)$. Then

$$
\int_{\Omega}\left[w_{\gamma}^{\prime}, w_{\gamma}\right] \phi d \Omega=\int_{\Omega}\left[\phi, w_{\gamma}\right] w_{\gamma}^{\prime} d \Omega
$$

and

$$
\frac{\partial^{2}}{\partial x_{i} \partial x_{j}} \phi \in H^{1-\epsilon}(\Omega) \subset C(\Omega) \text { for } i, j=1,2 .
$$

By Hölder's Inequality, we have

$$
\left\|\left[\phi, w_{\gamma}\right]\right\|_{C\left(0, T ; L_{2}(\Omega)\right)} \leq C
$$

Combining this result with our hypothesis, (2.56), we find

$$
\sup _{0 \leq t \leq T} \int_{\Omega}\left|\left[\phi, w_{\gamma}\right] w_{\gamma}^{\prime}\right| d \Omega \leq\left\|\left[\phi, w_{\gamma}\right]\right\|_{C\left(0, T ; L_{2}(\Omega)\right)}\left\|w_{\gamma}^{\prime}\right\|_{C\left(0, T ; L_{2}(\Omega)\right)} \leq C,
$$

so that

$$
\left\|\left[w_{\gamma}^{\prime}, w_{\gamma}\right]\right\|_{C\left(0, T ;\left[H^{3+\epsilon}(\Omega) \cap H_{0}^{2}(\Omega)\right]^{\prime}\right)} \leq C .
$$


By the compactness results of Grisvard, [?], and duality, we have

$$
\left[H^{3+\epsilon}(\Omega) \cap H_{0}^{2}(\Omega)\right]^{\prime} \sim\left[\mathcal{D}\left(\mathcal{K}^{\frac{3+\epsilon}{4}}\right)\right]^{\prime}
$$

where duality is with respect to the $L_{2}$-inner product. Now using $(2.61),(2.67)$, and (2.68), we obtain

$$
\begin{aligned}
\left\|\frac{\partial}{\partial t} \chi\left(w_{\gamma}\right)\right\|_{C\left(0, T ; \mathcal{D}\left(\mathcal{K}^{\left.\left.\frac{1-\epsilon}{4}\right)\right)}\right.\right.} & =\left\|\mathcal{K}^{\frac{1-\epsilon}{4}} \mathcal{K}^{-1}\left[w_{\gamma}^{\prime}, w_{\gamma}\right]\right\|_{C\left(0, T ; L_{2}(\Omega)\right)} \\
& =C\left\|\left[w_{\gamma}^{\prime}, w_{\gamma}\right]\right\|_{C\left(0, T ;\left[D\left(\mathcal{K}^{\frac{3+\epsilon}{4}}\right)\right]^{\prime}\right)} \leq \bar{C}
\end{aligned}
$$

This implies that

$$
\left\|\frac{\partial}{\partial t} \chi\left(w_{\gamma}\right)\right\|_{C\left(0, T ; H^{1-\epsilon}(\Omega)\right)} \leq C
$$

Using the compactness results proven by Simon, [?], we conclude that

$$
\chi\left(w_{\gamma}\right) \longrightarrow z \text { in } C\left(0, T ; H^{3-\epsilon}(\Omega)\right)
$$

Finally, using the result of Lemma 2.5 together with our assumptions, we conclude that $z=\chi(w)$, which completes the proof of Lemma 2.8 .

We may now pass with the limit as $\gamma \rightarrow 0$ in the equation for $v_{\gamma}$. Proceeding as in the previous argument (cases 1 and 2), we obtain analagous results and the remainder of the proof of Lemma 2.4 follows.

Combining the results of Proposition 2.1, (2.26), and Lemma 2.4, we obtain the desired result of Lemma 2.1, (2.1). By the same nonlinear semigroup argument as in section 2.3 of [?], we obtain (1.10) and the proof of Theorem 1.2 is complete.

\subsection{Proof of Corollary 1.1}

Corollary 1.1 follows directly from (1.10) by using the same argument as in the proof of Corollary 1.1 in [?], combined with the convergence results for $w_{\gamma}$ proven for the compactness/uniqueness argument in section 2.3 . 


\section{$3 \quad$ Proof of Lemma 2.3}

Step 1: Let $\psi(t) \in C_{0}^{\infty}(R)$ be a cutoff function defined such that $0 \leq \psi(t) \leq 1 \forall t$ and

$$
\psi(t)=\left\{\begin{array}{cc}
1 & \text { in }(\alpha, T-\alpha) \\
0 & \text { outside }\left(\frac{\alpha}{2}, T-\frac{\alpha}{2}\right) .
\end{array}\right.
$$

Define $w_{c}(t, \cdot) \equiv \psi(t) w(t, \cdot)$, where $w(t, \cdot)$ is our original solution to $(1.1)$. Then $w_{c}(t, \cdot)$ satisfies

$$
\begin{aligned}
& w_{c, t t}-\gamma^{2} \Delta w_{c, t t}+\Delta^{2} w_{c}=[\tilde{P}, \psi] w+\psi f \quad \text { in } Q_{\infty} \\
& \left.\begin{array}{l}
w_{c}(0)=0 \\
w_{c, t}(0)=0
\end{array}\right\} \quad \text { in } \Omega \\
& w_{e}=0 \quad \text { on } \Sigma_{\infty} \\
& \Delta w_{c}-(1-\mu) k \frac{\partial}{\partial \nu} w_{c}=-\psi \frac{\partial}{\partial \nu} w_{t} \quad \text { on } \Sigma_{\infty}
\end{aligned}
$$

where $[A, B]$ denotes the commutator of two operators, $A$ and $B$ and, in our case, $\tilde{P} w=w_{t t}-\gamma \Delta w_{t t}+\Delta^{2} w$. Rewriting this equation for $w_{c}$ as

$$
\begin{aligned}
& w_{c, t t}+\left(I-\gamma^{2} \Delta\right)^{-1} \Delta^{2} w_{c}=\left(I-\gamma^{2} \Delta\right)^{-1}[\tilde{P}, \psi] w+\left(I-\gamma^{2} \Delta\right)^{-1} \psi f \quad \text { in } Q_{\infty} \\
& \left.\begin{array}{l}
w_{c}(0)=0 \\
w_{c, t}(0)=0
\end{array}\right\} \quad \text { in } \Omega \\
& w_{c}=0 \quad \text { on } \Sigma_{\infty} \\
& \Delta w_{c}-(1-\mu) k \frac{\partial}{\partial \nu} w_{c}=-\psi \frac{\partial}{\partial \nu} w_{t} \quad \text { on } \Sigma_{\infty}
\end{aligned}
$$

we see that $w_{c}(t, \cdot)$ can be written implicitly using the variation of parameters formula. Therefore,

$$
w_{c}(t)=\frac{1}{2 i}{\sqrt{A_{\gamma}}}^{-1} \int_{0}^{t}\left(e^{i \sqrt{A_{\gamma}}(t-\tau)}-e^{-i \sqrt{A_{\gamma}}(t-\tau)}\right) F(\tau) d \tau
$$

where

$$
\begin{gathered}
A h \equiv \Delta^{2} h, \quad \mathcal{D}(A)=\left\{h \in H^{4}(\Omega) \cap H_{0}^{1}(\Omega):\left.\Delta h\right|_{\Gamma}=0\right\} \\
A_{\gamma} \equiv\left(I+\gamma^{2} A^{1 / 2}\right)^{-1} A \\
F(t) \equiv\left(I+\gamma^{2} A^{1 / 2}\right)^{-1}\left\{A^{1 / 2} D\left((1-\mu) k \frac{\partial}{\partial \nu} w_{c}-\psi \frac{\partial}{\partial \nu} w_{t}(t)\right)+[\tilde{P}, \psi] w(t)+\psi f\right\},
\end{gathered}
$$


and $D g$ is defined to be the harmonic extension of the function $g$ from the boundary into the interior, i.e.,

$$
D g=v \Longleftrightarrow\left\{\begin{aligned}
\Delta v=0 & \text { in } \Omega \\
v=g & \text { on } \Gamma .
\end{aligned}\right.
$$

Define

$$
\begin{gathered}
w_{1}(t) \equiv \frac{1}{2 i}{\sqrt{A_{\gamma}}}^{-1} \int_{0}^{t}\left(e^{i \sqrt{A_{\gamma}}(t-\tau)}-e^{-i \sqrt{A_{\gamma}}(t-\tau)}\right)\left(I+\gamma^{2} A^{1 / 2}\right)^{-1} \psi f(\tau) d \tau, \\
w_{2}(t) \equiv \frac{1}{2 i}{\sqrt{A_{\gamma}}}^{-1} \int_{0}^{t}\left(e^{i \sqrt{A_{\gamma}}(t-\tau)}-e^{-i \sqrt{A_{\gamma}}(t-\tau)}\right)\left(I+\gamma^{2} A^{1 / 2}\right)^{-1}\left\{A^{1 / 2} D\left((1-\mu) k \frac{\partial}{\partial \nu} w_{c}-\psi \frac{\partial}{\partial \nu} w_{t}(\tau)\right)\right\} d \tau, \\
w_{3}(t) \equiv \frac{1}{2 i}{\sqrt{A_{\gamma}}}^{-1} \int_{0}^{t}\left(e^{i \sqrt{A_{\gamma}}(t-\tau)}-e^{-i \sqrt{A_{\gamma}}(t-\tau)}\right)\left(I+\gamma^{2} A^{1 / 2}\right)^{-1}[\tilde{P}, \psi] w(\tau) d \tau .
\end{gathered}
$$

Step 2: Expressions for $w_{1}(t), w_{2}(t)$ and $w_{3}(t)$. Since $w_{c}$ is compactly supported on $(0, T)$,

$$
\left\|w_{c}\right\|_{H^{-1}(0, T)}=\left\|\int_{0}^{t} w_{c}(\tau) d \tau\right\|_{L_{2}(0, T)} .
$$

Hence,

$$
\left\|\frac{\partial}{\partial \nu} \Delta w_{c}\right\|_{H^{-1}\left(0, \infty ; L_{2}(\Gamma)\right)}=\left\|\frac{\partial}{\partial \nu} A^{1 / 2} \int_{0}^{t}\left(w_{1}+w_{2}+w_{3}\right)(\tau) d \tau\right\|_{L_{2}(\Sigma \infty)}
$$

Integrating $w_{1}(t)$ from 0 to $t$ gives

$$
\begin{aligned}
\frac{\partial}{\partial \nu} A^{1 / 2} \int_{0}^{t} w_{1}(\tau) d \tau \\
\quad=-\frac{1}{2} \frac{\partial}{\partial \nu} A^{-1 / 2} \int_{0}^{t}\left(e^{i \sqrt{A_{\gamma}}(t-\tau)}+e^{-i \sqrt{A_{\gamma}}(t-\tau)}-2\right) \psi f(\tau) d \tau
\end{aligned}
$$

Differentiating $w_{1}(t)$ with respect to $t$, we find

$$
w_{1}^{\prime}(t)=\frac{1}{2}\left(I+\gamma^{2} A^{1 / 2}\right)^{-1} \int_{0}^{t}\left(e^{i \sqrt{A_{\gamma}}(t-\tau)}+e^{-i \sqrt{A_{\gamma}}(t-\tau)}\right) \psi f(\tau) d \tau
$$

By noting that

$$
A^{1 / 2} A_{\gamma}^{-1}\left(I+\gamma^{2} A^{1 / 2}\right)^{-1} A^{1 / 2}=I,
$$

and

$$
\gamma^{2} A^{1 / 2}\left(I+\gamma^{2} A^{1 / 2}\right)^{-1}-I=\left(I+\gamma^{2} A^{1 / 2}\right)^{-1},
$$

we can compare these two expressions and find that

$$
\begin{aligned}
\frac{\partial}{\partial \nu} A^{1 / 2} & \int_{0}^{t} w_{1}(\tau) d \tau \\
& =-\gamma^{2} \frac{\partial}{\partial \nu} w_{1}^{\prime}(t)+\frac{\partial}{\partial \nu} A^{-1 / 2} \int_{0}^{t} \psi f(\tau) d \tau \\
& +\frac{1}{2} \frac{\partial}{\partial \nu}\left(I+\gamma^{2} A^{1 / 2}\right)^{-1} A^{-1 / 2} \int_{0}^{t}\left(e^{i \sqrt{A_{\gamma}}(t-\tau)}+e^{-i \sqrt{A_{\gamma}}(t-\tau)}\right) \psi f(\tau) d \tau
\end{aligned}
$$


Proceeding in the same manner as for $w_{1}(t)$, we can find corresponding identities for $w_{2}(t)$ and $w_{3}(t)$ (see $[?, ?])$. Combining the resulting expressions with 3.14 , we find

$$
\begin{aligned}
& \left\|\frac{\partial}{\partial \nu} A^{1 / 2} w_{c}\right\|_{H^{-1}\left(0, \infty ; L_{2}(\Gamma)\right)} \\
& \leq \gamma^{2}\left\|\frac{\partial}{\partial \nu}\left(w_{c}\right)_{t}\right\|_{L_{2}\left(\Sigma_{\infty}\right)}+\left\|\frac{\partial}{\partial \nu} \int_{0}^{t} A^{-1 / 2} D\left(k \frac{\partial}{\partial \nu} w_{c}(\tau)\right) d \tau\right\|_{L_{2}(\Sigma \infty)} \\
& +\frac{1}{2}\left\|\frac{\partial}{\partial \nu} \int_{0}^{t}\left(e^{i \sqrt{A_{\gamma}}(t-\tau)}+e^{-i \sqrt{A_{\gamma}}(t-\tau)}\right)\left(I+\gamma^{2} A^{1 / 2}\right)^{-1} D\left(k \frac{\partial}{\partial \nu} w_{c}(\tau)\right) d \tau\right\|_{L_{2}\left(\Sigma_{\infty}\right)} \\
& +\left\|\frac{\partial}{\partial \nu} \int_{0}^{t} \psi(\tau) D\left(\frac{\partial}{\partial \nu} w_{t}(\tau)\right) d \tau\right\|_{L_{2}(\Sigma \infty)} \\
& +\frac{1}{2}\left\|\frac{\partial}{\partial \nu} \int_{0}^{t}\left(e^{i \sqrt{A_{\gamma}}(t-\tau)}+e^{-i \sqrt{A_{\gamma}}(t-\tau)}\right)\left(I+\gamma^{2} A^{1 / 2}\right)^{-1} \psi(\tau) D\left(\frac{\partial}{\partial \nu} w_{t}(\tau)\right) d \tau\right\|_{L_{2}\left(\Sigma_{\infty}\right)} \\
& +\left\|\frac{\partial}{\partial \nu} \int_{0}^{t} A^{-1 / 2}[\tilde{P}, \psi] w(\tau) d \tau\right\|_{L_{2}(\Sigma \infty)} \\
& +\frac{1}{2}\left\|\frac{\partial}{\partial \nu} \int_{0}^{t}\left(e^{i \sqrt{A_{\gamma}}(t-\tau)}+e^{-i \sqrt{A_{\gamma}}(t-\tau)}\right)\left(I+\gamma^{2} A^{1 / 2}\right)^{-1} A^{-1 / 2}[\tilde{P}, \psi] w(\tau) d \tau\right\|_{L_{2}\left(\Sigma_{\infty}\right)} \\
& +\left\|\frac{\partial}{\partial \nu} \int_{0}^{t} A^{-1 / 2} \psi f(\tau) d \tau\right\|_{L_{2}\left(\Sigma_{\infty}\right)} \\
& +\frac{1}{2}\left\|\frac{\partial}{\partial \nu} \int_{0}^{t}\left(e^{i \sqrt{A_{\gamma}}(t-\tau)}+e^{-i \sqrt{A_{\gamma}}(t-\tau)}\right)\left(I+\gamma^{2} A^{1 / 2}\right)^{-1} A^{-1 / 2} \psi f(\tau) d \tau\right\|_{L_{2}\left(\Sigma_{\infty}\right)} .
\end{aligned}
$$

From [?] and [?], we know terms two through seven on the right-hand side of (3.15) are bounded by:

$$
C_{T}\left(1+\gamma^{2}\right)\left\|\frac{\partial}{\partial \nu} w_{t}\right\|_{L_{2}\left(\Sigma_{T}\right)}^{2}+C(T+1)\left(1+\gamma^{2}\right)\|w\|_{L_{2}\left(0, T ; H^{2-\epsilon}(\Omega)\right)}^{2}
$$

hence, we need to focus our attention on the last two terms on the right-hand side of (3.15). To bound the last term, we will use the following sharp regularity result for an abstract form of the Schrödinger equation which was proven in [?].

Proposition 3.1 Consider the following "abstract" Schrödinger problem:

$$
\begin{gathered}
z_{t}=i \sqrt{A_{\gamma}} z+\left(I+\gamma A^{1 / 2}\right)^{-1} f \\
z(0)=0
\end{gathered}
$$

Then if $z(t)$ satisfies (3.17),

$$
\left\|\frac{\partial}{\partial \nu} z\right\|_{L_{2}\left(\Sigma_{T}\right)} \leq C(T+1)\left\|A^{1 / 4}\left(I+\gamma A^{1 / 2}\right)^{-1} f\right\|_{L_{1}\left(0, T ; L_{2}(\Omega)\right)}
$$

which in turn implies

$$
\left\|\frac{\partial}{\partial \nu} z\right\|_{L_{2}\left(\Sigma_{T}\right)} \leq C(T+1)\|f\|_{L_{1}\left(0, T ; H_{0}^{1}(\Omega)\right)}
$$

where the constant $C$ is independent of $\gamma$. 
Applying Proposition 3.1 to the last term on the right-hand side of (3.15), we find

$$
\begin{aligned}
\| \frac{\partial}{\partial \nu} \int_{0}^{t}\left(e^{i \sqrt{A_{\gamma}}(t-\tau)}\right. & \left.+e^{-i \sqrt{A_{\gamma}}(t-\tau)}\right)\left(I+\gamma^{2} A^{1 / 2}\right)^{-1} A^{-1 / 2} \psi f(\tau) d \tau \|_{L_{2}\left(\Sigma_{\infty}\right)} \\
& \leq C(T+1)\left\|A^{-1 / 2} \psi f\right\|_{L_{1}\left(0, \infty ; H_{0}^{1}(\Omega)\right)} \\
& \leq C(T+1)\|f\|_{L_{1}\left(0, T ; H^{-1}(\Omega)\right)} \leq C(T+1)\|f\|_{L_{2}\left(0, T ; H^{-1}(\Omega)\right)}
\end{aligned}
$$

The second to last term on the right-hand side of (3.15) may be bounded directly using trace theory:

$$
\left\|\frac{\partial}{\partial \nu} \int_{0}^{t} A^{-1 / 2} \psi f(\tau) d \tau\right\|_{L_{2}\left(\Sigma_{\infty}\right)} \leq C\left\|A^{-1 / 2} \psi f\right\|_{H^{-1}\left(0, \infty ; H^{3 / 2+\epsilon}(\Omega)\right)} \leq C\|f\|_{L_{2}\left(0, T ; H^{-1 / 2+\epsilon}(\Omega)\right)}
$$

Substituting (3.16), (3.20), and (3.21) into (3.15), we find

$$
\begin{gathered}
\left\|\frac{\partial}{\partial \nu} A^{1 / 2} w_{\epsilon}\right\|_{H^{-1}\left(0, \infty ; L_{2}(\Gamma)\right)} \leq C_{T}\left(1+\gamma^{2}\right)\left\|\frac{\partial}{\partial \nu} w_{t}\right\|_{L_{2}\left(\Sigma_{T}\right)}^{2}+C(T+1)\left(1+\gamma^{2}\right)\|w\|_{L_{2}\left(0, T ; H^{2-\epsilon}(\Omega)\right)}^{2} \\
+C\|f\|_{L_{2}\left(0, T ; H^{-1 / 2+\epsilon}(\Omega)\right)},
\end{gathered}
$$

our desired result. 